\title{
Clinical outcomes of diffuse axonal injury after traumatic brain injury according to magnetic resonance grading
}

\author{
Insu Lee ${ }^{1}$, Kawngwoo Park ${ }^{1}$, Tae Seok Jeong ${ }^{1}$, Woo-Seok Kim ${ }^{1}$, Woo Kyung Kim ${ }^{1}$, Do Yeon Rhee ${ }^{1}$, Cheol Wan Park ${ }^{2}$ \\ ${ }^{1}$ Department of Neurosurgery, Gachon University Gil Medical Center, Gachon University College of Medicine, Incheon, Korea \\ ${ }^{2}$ Department of Emergency Medicine, Gachon University Gil Medical Center, Gachon University College of Medicine, Incheon, Korea
}

Objective: Diffuse axonal injury (DAl) is a severe form of traumatic brain injury (TBI) caused by shearing damage to the axons of long connecting fibers. In this study, we evaluated the clinical features according to DAl grading and identified prognostic factors.

Methods: The study involved patients diagnosed with DAl using magnetic resonance imaging within 2 weeks of being hospitalized for TBI from January 2019 to December 2019. Patients who received surgical treatments, such as craniectomy or craniotomy for hematoma evacuation or an external ventricular drain, were excluded. To determine prognostic factors, we retrospectively reviewed the demographic, clinical, and radiological characteristics of 41 patients. Patients' outcomes were analyzed using the Glasgow Outcome Scale within 6 months of the traumatic injury.

Results: Using a multivariate logistic regression model, unfavorable outcomes were significantly associated with lower Glasgow Coma Scale score ( $P=0.03)$, DAl grade III $(P=0.03)$, DAl located at the stem $(P=0.04)$, and the presence of 7 or more DAls $(P=0.03)$. However, $\mathrm{DAl}$ grade II was not a significant prognostic factor $(\mathrm{P}=0.76)$. Furthermore, the rates of hospital-acquired infection $(\mathrm{P}=0.04)$ and tracheostomy $(\mathrm{P}=0.03)$ significantly increased according to $\mathrm{DAl}$ grade.

Conclusion: Our results provide valuable information on prognostic predictors in patients with TBI.

Keywords: Diffuse axonal injury; Traumatic brain injury; Brain magnetic resonance imaging; Prognosis

\section{Introduction}

Diffuse axonal injury (DAI) occurs in approximately $40 \%$ to $50 \%$ of patients with severe traumatic brain injury (TBI) [1-4]. DAI is a shear injury caused by mechanical force and occurs as axial damage $[5,6]$. DAI contributes to increasing the duration of unconsciousness, mortality, and disability in patients with TBI $[2,7]$. Identifying DAI in patients with TBI helps predict the recovery of consciousness and clinical outcomes [2-4,8]. Furthermore, patients with DAI are often accompanied by damage in other body parts and mixed with several forms of intracranial hemorrhage in a complicated state. For these reasons, the outcome of patients with

\footnotetext{
Received: December 4, 2020 Revised: December 10, 2020
}

Accepted: December 19, 2020

Corresponding author: Kawngwoo Park, MD, PhD

Department of Neurosurgery, Gachon University Gil Medical Center, 21

Namdong-daero 774beon-gil, Namdong-gu, Incheon 21565, Korea

Tel: +82-32-460-3304; Fax: +82-32-460-3899; E-mail: medicwoo@gilhospital.com
DAI is diverse, ranging from death to full recovery $[2,6,9,10]$. Currently, with the advances in neurological imaging, especially brain magnetic resonance imaging (MRI), DAI can be more easily diagnosed, and DAI grading helps predict the prognosis [8,11-13].

However, controversial results were reported by some studies. Chelly et al. [1] have shown that MRI classification does not have a prognostic value. Moreover, Hedaoo et al. [14] have mentioned that MRI grading and the Glasgow Coma Scale (GCS) do not have a prognostic value.

Thus, this study was conducted to describe the clinical, epidemiological, and radiological features in patients with post-traumatic DAI; to examine the correlation between the severity of DAI based on MRI grading and clinical outcomes, time to consciousness recovery, and duration of intensive care unit stay; and to determine factors that could help predict prognosis. 


\section{Material and Methods}

\section{Patient selection}

The study involved patients with TBI admitted to the Neurosurgery Department via the Emergency Room (ER) at Gachon University Gil Medical Center. The study was approved by the Institutional Review Board of our institution (IRB No: GBIRB2020 -293). Informed consent was waived because of the retrospective nature of the study and the analysis used anonymous clinical data. Patients with TBI who were hospitalized from January 2019 to December 2019 were retrospectively reviewed using their medical records. Among them, 41 patients diagnosed with DAI from MRI within 2 weeks of admission were selected (Fig. 1). Patients who underwent surgical treatment, such as craniotomy/craniotomy for hematoma evacuation and external ventricular drain, those not diagnosed with DAI, and those not followed up 6 months after trauma were excluded.

The age and sex information of the patients was described, and the GCS score of each patient was recorded at the ER. The mechanical ventilation period, duration of intensive care unit (ICU) stay, initial hemoglobin level, lactate level, and the number of transfused red blood cell (RBC) packs during hospitalization were checked. Moreover, surgery due to accompanying damage to other body parts and anticoagulant intake were recorded. In addition,

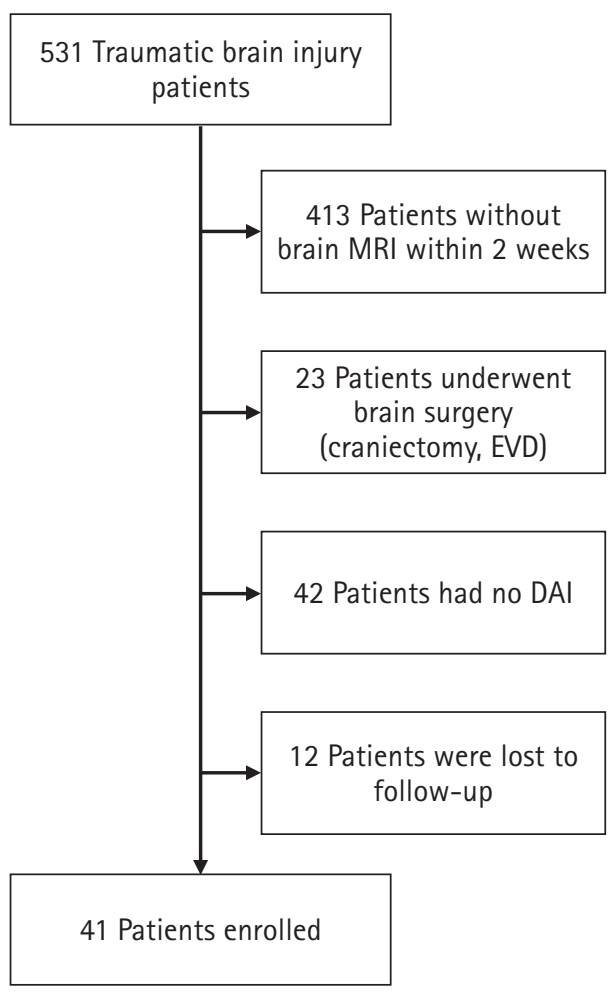

Fig. 1. Patient selection process. MRI, magnetic resonance imaging; EVD, external ventricular drain; DAI, diffuse axonal injury. tracheostomy application and hospital-acquired infection status were investigated. Lastly, Glasgow Outcome Scale (GOS) was checked 6 months after trauma. Based on the results, we divided the GOS outcomes into two groups: the favorable outcome (GOS 4,5 ) and unfavorable outcome (GOS 1-3) groups.

\section{Radiological strategy}

For all patients, computed tomography (CT) was performed in the ER within the first 24 hours, and only the initial CT was included in the study. Using CT images, the presence of intracranial, subdural, subarachnoid, epidural, intracerebral, and intraventricular hemorrhage were examined. To use brain CT as an objective indicator of TBI, the Marshall classification, Rotterdam CT score, and Helsinki score were used [15-17]. Using MRI, T2-weighted fast spin-echo, T2-weighted gradient echo, and susceptibility-weighted imaging were checked. In the case where DAI was found, the values were classified into grades I, II, and III according to the Gentry classification (Fig. 2) [18], which was cross-validated by the description of a neurosurgeon and radiologist for the accuracy of the diagnosis.

\section{Statistical analysis}

To determine the differences among factors for each grade, the chisquare test, Fisher exact, and one-way analysis of variance (Scheffe correction for post-hoc analysis) were performed. Furthermore, to assess the impact of DAI quantity on prognosis, the points with the highest sensitivity and specificity of more than or equal to 7 were set as the cut-off values through the receiver operating characteristic (ROC) curve (Fig. 3). To predict the outcomes and examine prognostic factors, we checked all features by performing univariate analysis. Factors identified by the univariate analysis were applied to a multivariate analysis using a binary logistic regression model. Statistical analysis was performed using SPSS ver. 25.0 (IBM Corp., Armonk, NY, USA).

\section{Results}

Among the patients with confirmed DAI, 19 (46\%) were grade I, 13 (32\%) were grade II, and nine (22\%) were grade III. Among the 41 patients, six were female, and 35 were male. The median age was 57 years (range, 29.5-63.0 years). At admission, the mean GCS score was 10.59. Moreover, the average mechanical ventilation period was 4.37 days, and the mean duration of ICU stay was 11.17 days. The mean lactate level at admission was $2.8 \mathrm{mmol} / \mathrm{L}$, and the mean hemoglobin level was $13.82 \mathrm{~g} / \mathrm{dL}$. The mean number of RBC packs transfused during the hospitalization period was 1.34. Among the 41 patients, seven (17\%) underwent tracheosto- 

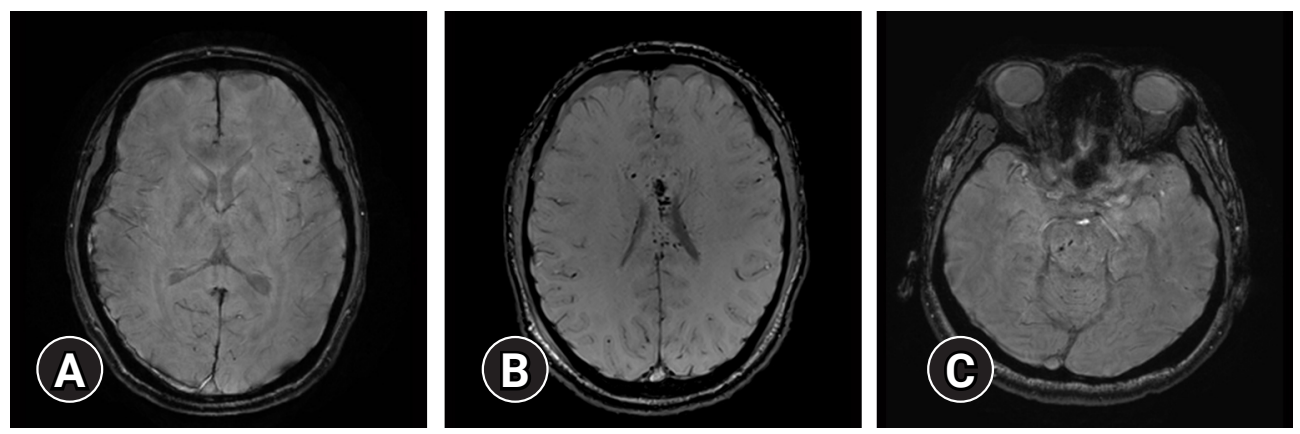

Fig. 2. Susceptibility-weighted imaging of patients with traumatic brain injury. (A) Small traumatic hemorrhage located in the subcortical white matter. (B) Multiple axonal hemorrhages from the corpus callosum body. (C) Axonal injury found from the brainstem.

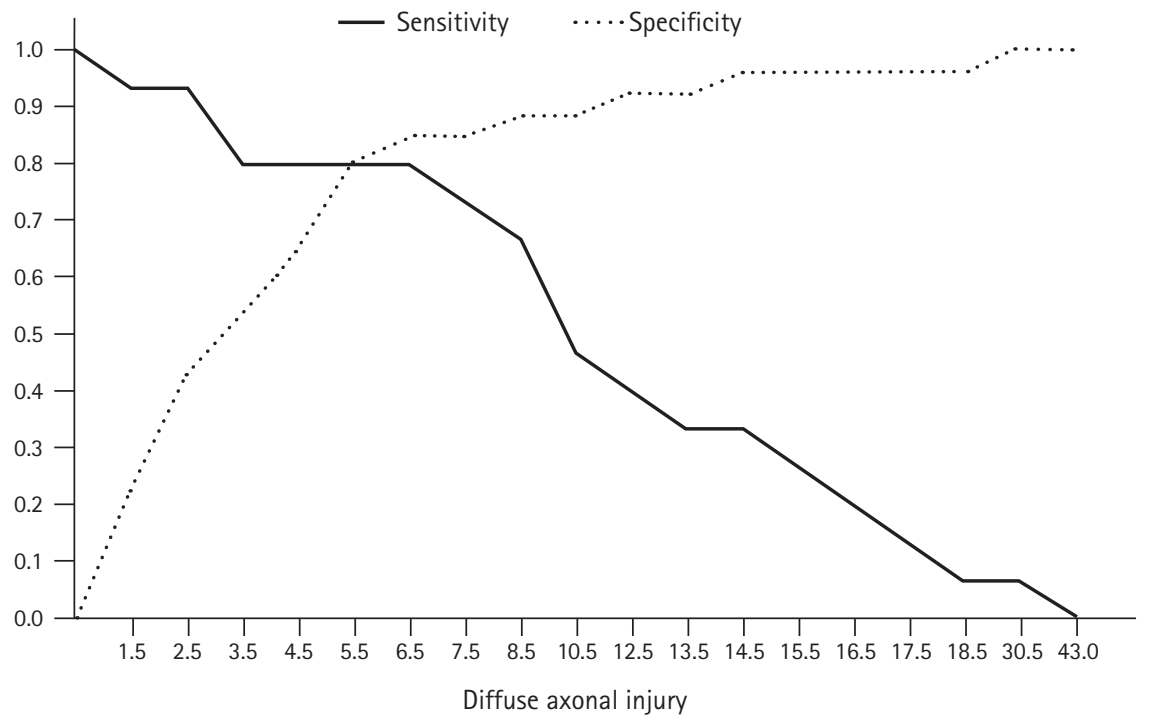

Fig. 3. Cut-off value of the number of DAls to predict unfavorable outcomes. Using an ROC curve, when the DAI number was 6.5, the sensitivity was 0.8 and specificity was 0.85 , leading to the greatest sum of predicted unfavorable outcomes. Based on this result, we set the cut-off value as greater than or equal to 7 to predict unfavorable outcomes. DAl, diffuse axonal injury; ROC, receiver operating characteristic.

my, and 11 (27\%) had hospital-acquired infection.

Analyzing the results, one patient (2\%) has taken an anticoagulant for cardiovascular disease. Eleven patients (27\%) underwent surgery for extracranial complications after trauma. Initial CT analysis revealed that 19 patients (46\%) had subdural hemorrhage, 22 (54\%) had subarachnoid hemorrhage, one (2\%) had epidural hemorrhage, four (10\%) had intracerebral hemorrhage, and nine (22\%) had intraventricular hemorrhage. No hemorrhage was detected in eight patients (20\%) (Table 1).

In the analysis of major trauma incidents, the injury resulted from the following mechanisms: 16 (39\%) resulted from a pedestrian traffic accident (TA), five (12\%) from passenger TA, two (5\%) from bicycle accidents, five (12\%) from motorbike accidents, and 13 (32\%) from falls (Table 2). DAI location and quantity confirmed from MRI scanning are summarized in Table 3.
The distribution of patients with unfavorable outcomes (GOS 1-3) was as follows: two were grade I, six were grade II, and seven were grade III. For the distribution of patients with favorable outcomes (GOS 4, 5) 17 were grade I, seven were grade II, and two were grade III (Fig. 4). The following variables were significantly different among grade I-III patients: median age $(\mathrm{P}=0.04)$, GCS score at admission $(\mathrm{P}=0.01)$, number of DAI $(\mathrm{P}<0.001)$, and duration of ICU stay $(\mathrm{P}=0.04)$ (Table 4$)$. As DAI grading increased, the rates of hospital-acquired infection and tracheostomy rose $(\mathrm{P}=0.04$ and $\mathrm{P}=0.03$, respectively) (Table 5$)$. To determine the prognostic factors related to unfavorable outcomes, we analyzed all characteristics of the factors. From the univariate analysis, we discerned significant factors and applied them to multivariate analysis (Table 6). We found that prognostic factors could be significant when having an initial GCS, DAI located at the stem, DAI 
grade III (compared with grade I), and DAI quantity of 7 or more.

\section{Discussion}

The most notable point from this study is that a significantly unfa-

Table 1. Clinical and demographic characteristics of patients

\begin{tabular}{lc}
\hline Characteristic & Value \\
\hline Age (yr) & $57(29.50-63.00)$ \\
Anticoagulant & $1(2)$ \\
Sex (male/female) & $35 / 6(85 / 15)$ \\
Hospital-acquired infection & $11(27)$ \\
Intensive care unit stay (day) & $11.17 \pm 18.59$ \\
Initial GCS & $10.59 \pm 4.00$ \\
Initial hemoglobin (g/dL) & $13.82 \pm 1.90$ \\
Initial lactate (mmol/L) & $2.80 \pm 2.30$ \\
Mechanical ventilation period & $4.37 \pm 13.98$ \\
Surgery for extracranial lesion & $11(27)$ \\
Tracheostomy & $7(17)$ \\
Transfusion (RBC packs) & $1.34 \pm 3.29$ \\
Initial brain CT findings & \\
Epidural hemorrhage & $1(2)$ \\
Intracerebral hemorrhage & $4(10)$ \\
Intraventricular hemorrhage & $9(22)$ \\
No hemorrhage & $8(20)$ \\
Subarachnoid hemorrhage & $22(54)$ \\
Subdural hemorrhage & $19(46)$ \\
DAI grade & \\
I & $19(46)$ \\
II & $13(32)$ \\
III & $9(22)$ \\
\hline
\end{tabular}

Values are presented as median (range), number (\%), or mean \pm standard deviation.

GCS, Glasgow Coma Scale; RBC, red blood cell; CT, computed tomography; DAl, diffuse axonal injury. vorable outcome is predicted when there is more than seven DAIs observed from MRI scans. We set 10 spots in the MRI scans and counted the number of patients with DAI in those spots. Furthermore, we calculated the DAI quantity; found that the highest point with specificity and sensitivity from the ROC curve is 6.5; and set the cut-off value to more or equal to 7 . If DAI is more or equal to 7 , it had a significant effect on the unfavorable outcome (odds ratio

Table 2. Mechanism of injury in diffuse axonal injury patients

\begin{tabular}{lc}
\hline Type & No. $(\%)$ \\
\hline Bicycle & $2(5)$ \\
Fall down & $13(32)$ \\
Motorcycle & $5(12)$ \\
Passenger traffic accident & $5(12)$ \\
Pedestrian traffic accident & $16(39)$ \\
\hline
\end{tabular}

Table 3. Location of DAI on magnetic resonance imaging

\begin{tabular}{lc}
\hline Location & No. $(\%)$ \\
\hline Centrum semiovale & $12(29)$ \\
Cerebellum & $9(22)$ \\
Corpus callosum & $19(46)$ \\
$\quad$ Splenium & $7(17)$ \\
Genu & $8(20)$ \\
$\quad$ Body & $15(37)$ \\
Deep gray matter & $15(37)$ \\
$\quad$ Lentiform nucleus & $8(20)$ \\
Caudate nucleus & $3(7)$ \\
$\quad$ Thalamus & $8(20)$ \\
Stem & $9(22)$ \\
Subcortical white matter & $29(71)$ \\
Quantity of DAls (mean \pm SD) & $7.27 \pm 7.72$
\end{tabular}

DAl, diffuse axonal injury; SD, standard deviation.

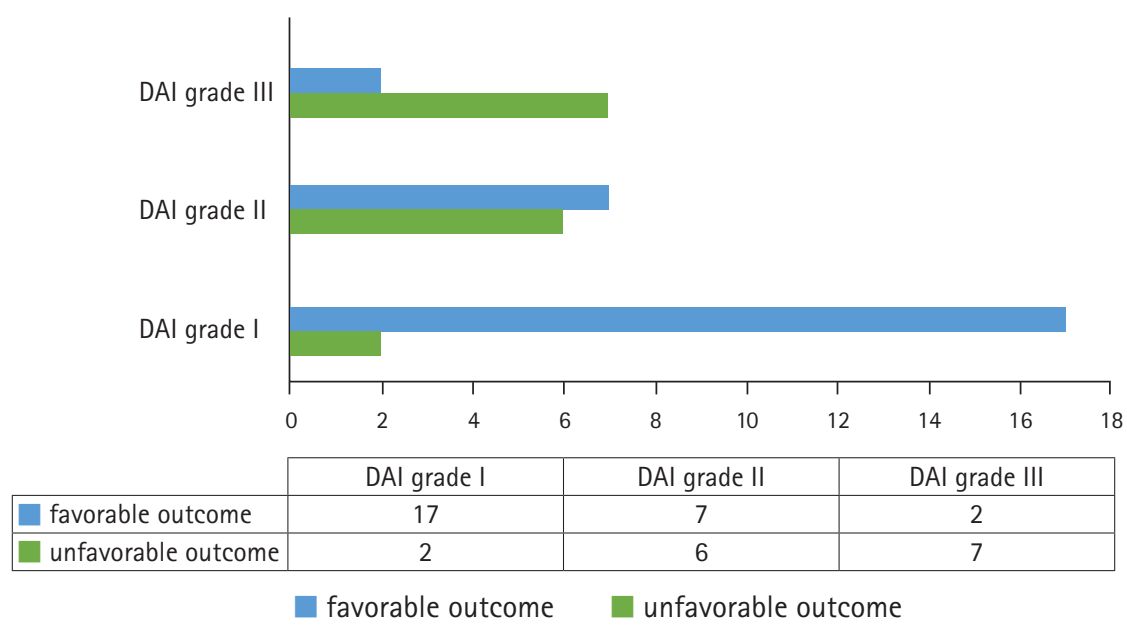

Fig. 4. Clinical outcomes of diffuse axonal injury (DAI) patients according to grading. 
Table 4. Clinical features of DAl patients by magnetic resonance imaging grading

\begin{tabular}{lcccc}
\hline Variable & DAl grade I $(n=19)$ & DAl grade II $(n=13)$ & DAl grade III $(n=9)$ & P-value \\
\hline Age (yr) & 56.58 & 39.46 & 49.89 & $0.04^{*}$ \\
Duration of ICU stay (day) & 3.74 & 16.23 & 19.56 & $0.04^{*}$ \\
Duration of mechanical ventilation (day) & 0.11 & 5.31 & 12.00 & 0.10 \\
Male sex (\%) & 84 & 77 & 100 & 0.33 \\
GCS at admission & 12.58 & 9.00 & 8.67 & $0.01^{*}$ \\
Initial hemoglobin (g/dL) & 13.63 & 14.25 & 13.62 & 0.63 \\
Initial lactate (mmoI/L) & 2.41 & 2.86 & 3.54 & 0.48 \\
Marshal classification (grade) & 1.89 & 2.15 & 1.89 & 0.50 \\
Number of DAls & 2.89 & 12.31 & 9.22 & $<0.001^{*}$ \\
Transfusion (RBC packs) & 0.63 & 1.85 & 2.11 & 0.44
\end{tabular}

DAI, diffuse axonal injury; ICU, intensive care unit; GCS, Glasgow Coma Scale; RBC, red blood cell.

*Statistically significant differences $(\mathrm{P}<0.05)$.

Table 5. Clinical features of DAl patients by magnetic resonance imaging grading

\begin{tabular}{lcccc}
\hline Variable & DAl grade I $(n=19)$ & DAl grade II $(n=13)$ & DAl grade III $(n=9)$ & P-value \\
\hline Anticoagulant for cardiovascular disease & $1(5)$ & 0 & 0 & 0.55 \\
Application of tracheostomy & 0 & $4(31)$ & $3(33)$ & $0.03^{*}$ \\
Hospital-acquired infection & $2(11)$ & $4(31)$ & $5(56)$ & $0.04^{*}$ \\
Surgery for an extracranial lesion & $2(11)$ & $5(38)$ & $4(44)$ & 0.09 \\
\hline
\end{tabular}

Values are presented as number (\%).

DAl, diffuse axonal injury.

*Statistically significant differences $(\mathrm{P}<0.05)$.

Table 6. Prognostic factors associated with unfavorable outcomes (multivariate regression analysis)

\begin{tabular}{lcc}
\hline Factor & Odds ratio (95\% Cl) & P-value \\
\hline DAl grade & & \\
$\quad$ Grade II & $0.64(0.039-10.56)$ & 0.76 \\
$\quad$ Grade III & $13.99(0.86-293.86)$ & $0.03^{*}$ \\
DAl location & & \\
$\quad$ Centrum semiovale & $6.83(0.44-105.44)$ & 0.17 \\
$\quad$ Corpus callosum (splenium) & $2.77(0.14-54.20)$ & 0.50 \\
$\quad$ Stem & $18.19(1.20-276.51)$ & $0.04^{*}$ \\
DAl quantity $\geq 7$ & $10.86(1.33-88.55)$ & $0.03^{*}$ \\
Initial GCS & $0.64(0.43-0.95)$ & $0.03^{*}$ \\
\hline
\end{tabular}

$\mathrm{Cl}$, confidence interval; DAl, diffuse axonal injury; GCS, Glasgow Coma Scale.

*Statistically significant differences $(\mathrm{P}<0.05)$.

[OR], 10.86; P = 0.03). Chelly et al. [1] have shown that mortality increases when the DAI count is greater or equal to 6 , which is related to poor outcomes assessed using the GOS. Similar findings were observed in this study. Based on the results, we suggest that the DAI count has a prognostic value, in addition to the GCS and DAI grading, which were proven as significant factors $[6,7]$. This suggests that brain injury is multiple and is considered related to unfavorable outcomes.
Furthermore, among the 10 spots, those that significantly affected unfavorable outcomes were the stem, splenium, and centrum semiovale from univariate analysis, and those located at stem from multivariate regression analysis were considered significant (OR, $18.19 ; \mathrm{P}=0.04$ ). We suppose that the extensive scale of damage plays a more significant role than the specific location of the injury. Aside from the aforementioned features, factors that showed significance were DAI grade III (OR, 13.99; $\mathrm{P}=0.03)$ and initial GCS (OR, 0.64; $\mathrm{P}=0.03$ ). DAI grade II had no significance $(\mathrm{P}=0.76)$. The hemorrhage types from the initial CT were not significant.

Referring to other research results, van Eijck et al. [6] have reported that the probability of having unfavorable outcomes rises as MRI grading increases. Skandsen et al. [11] have shown that the results would not be significant with grade II and that it would be significant as a prognostic factor only with grade III. This corresponded with the results of this study.

Additionally, we checked the differences in clinical features according to each DAI grade. As a result, significant differences in age $(\mathrm{P}=0.04)$, initial GCS $(\mathrm{P}=0.01)$, DAI quantity $(\mathrm{P}<0.001)$, and duration of ICU stay $(\mathrm{P}=0.04)$ were observed. Moreover, a difference in the rate of tracheostomy $(\mathrm{P}=0.03)$ and hospital-acquired infection $(\mathrm{P}=0.04)$ was observed according to DAI grad- 
ing. In the study by Lee et al. [10], the tracheostomy rate increased according to DAI grading, which is similar to the results of this study.

However, this study has some limitations. First, this is a retrospective study conducted at a single-center, involving a small patient population. MRI was not performed in all patients, and the time brain MRI was performed for each patient was different. Moreover, we estimated the cut-off value by selecting the DAI count for which the sum of sensitivity and specificity is the highest, using the ROC curve. As the focus could be on either sensitivity or specificity, the setpoint could be different, and this may cause a change in the results. Another limitation is that we excluded patients who underwent surgery. As a regional trauma center, our medical center has several patients who need surgical treatment with high severity of illness. Including patients with higher severity than the entire patient group could be a limitation in this study. Nonetheless, we confirmed that the DAI quantity has a prognostic value. By doing a follow-up study, we look forward to proving this statement further.

\section{Conclusion}

In this study, when DAI quantity was greater or equal to 7 on MRI, an effect with an unfavorable outcome occurs. Furthermore, the initial GCS, MRI grading III, and DAI location at the stem had a significant effect on the outcome. The study offers findings that may help physicians predict the prognosis in patients with TBI.

\section{Conflicts of interest}

No potential conflict of interest relevant to this article was reported.

\section{ORCID}

Insu Lee, https://orcid.org/0000-0003-2509-8131

Kawngwoo Park, https://orcid.org/0000-0002-6568-1009

\section{REFERENCES}

1. Chelly H, Chaari A, Daoud E, et al. Diffuse axonal injury in patients with head injuries: an epidemiologic and prognosis study of 124 cases. J Trauma 2011;71:838-46.

2. Paterakis K, Karantanas AH, Komnos A, Volikas Z. Outcome of patients with diffuse axonal injury: the significance and prognostic value of MRI in the acute phase. J Trauma 2000;49:1071-5.

3. Eum SW, Lim DJ, Kim BR, et al. Prognostic factors in patients with diffuse axonal injury. J Korean Neurosurg Soc 1998;27: 1668-74.

4. NIH Consensus Development Panel on Rehabilitation of Persons with Traumatic Brain Injury. Rehabilitation of persons with traumatic brain injury.JAMA 1999;282:974-83.

5. Meythaler JM, Peduzzi JD, Eleftheriou E, Novack TA. Current concepts: diffuse axonal injury-associated traumatic brain injury. Arch Phys Med Rehabil 2001;82:1461-71.

6. van Eijck MM, Schoonman GG, van der Naalt J, de Vries J, Roks G. Diffuse axonal injury after traumatic brain injury is a prognostic factor for functional outcome: a systematic review and meta-analysis. Brain Inj 2018;32:395-402.

7. Wallesch CW, Curio N, Kutz S, Jost S, Bartels C, Synowitz H. Outcome after mild-to-moderate blunt head injury: effects of focal lesions and diffuse axonal injury. Brain Inj 2001;15:401-12.

8. Humble SS, Wilson LD, Wang L, et al. Prognosis of diffuse axonal injury with traumatic brain injury. J Trauma Acute Care Surg 2018;85:155-9.

9. Park SJ, Hur JW, Kwon KY, Rhee JJ, Lee JW, Lee HK. Time to recover consciousness in patients with diffuse axonal injury : assessment with reference to magnetic resonance grading. J Korean Neurosurg Soc 2009;46:205-9.

10. Lee HJ, Sun HW, Lee JS, Choi NJ, Jung YJ, Hong SK. Clinical outcomes of diffuse axonal injury according to radiological grade. J Korean Soc Traumatol 2018;31:51-7.

11. Skandsen T, Kvistad KA, Solheim O, Strand IH, Folvik M, VikA. Prevalence and impact of diffuse axonal injury in patients with moderate and severe head injury: a cohort study of early magnetic resonance imaging findings and 1-year outcome. J Neurosurg 2010;113:556-63.

12. Alberico AM, Ward JD, Choi SC, Marmarou A, Young HF. Outcome after severe head injury: relationship to mass lesions, diffuse injury, and ICP course in pediatric and adult patients. J Neurosurg 1987;67:648-56.

13. Vieira RC, Paiva WS, de Oliveira DV, Teixeira MJ, de Andrade AF, de Sousa RM. Diffuse axonal injury: epidemiology, outcome and associated risk factors. Front Neurol 2016;7:178.

14. Hedaoo K, Rajpurohit V, Garg S, Thanvi S, Nagocha VB. GCS score and MRI grading does not predicts the outcome in DAI patients: a prospective study. Romanian Neurosurg 2018;32: 314-21.

15. Maas AI, Hukkelhoven CW, Marshall LF, Steyerberg EW. Prediction of outcome in traumatic brain injury with computed tomographic characteristics: a comparison between the computed tomographic classification and combinations of computed tomographic predictors. Neurosurgery 2005;57:1173-82.

16. Raj R, Siironen J, Skrifvars MB, Hernesniemi J, Kivisaari R. Pre- 
dicting outcome in traumatic brain injury: development of a novel computerized tomography classification system (Helsinki computerized tomography score). Neurosurgery 2014;75:63246.

17. Marshall LF, Marshall SB, Klauber MR, et al. A new classification of head injury based on computerized tomography. J Neurosurg 1991;75:S14-20.

18. Gentry LR. Imaging of closed head injury. Radiology 1994; 191:1-17. 